\title{
Correction to: Biomethanol Production from Methane by Immobilized Co-cultures of Methanotrophs
}

\author{
Sanjay K. S. Patel ${ }^{1} \cdot$ Rahul K. Gupta $^{1} \cdot$ Virendra Kumar $^{1} \cdot$ Sanath Kondaveeti $^{1} \cdot$ Anurag Kumar $^{1}$ • \\ Devashish Das ${ }^{1} \cdot$ Vipin Chandra Kalia $^{1} \cdot$ Jung-Kul Lee $^{1}$
}

Published online: 21 May 2021

(C) Association of Microbiologists of India 2021

\section{Correction to:}

Indian J Microbiol (July-Sept 2020) 60(3):318-324 https://doi.org/10.1007/s12088-020-00883-6

The original version of this article was published on 22 May 2020 in Volume 60, issue 3, page 318-324, contained a mistake in the year of the Acknowledgements.

It reads as:

"Acknowledgements This work was supported by Brain Pool grant (NRF-2019H1D3A201060226) by National Research Foundation of Korea (NRF) to work at Konkuk University. This research was sup-ported by Basic Science Research Program through the NRF funded by the Ministry of Science, ICT \& Future Planning (NRF2020H1D3A2A01060467, NRF-2019R1C1C11009766).
This work was also supported by KU Research Professor program of Konkuk University. This paper was supported by Konkuk University Researcher Fund in 2018.”

It should read as:

"Acknowledgements This work was supported by Brain Pool grant (NRF-2019H1D3A201060226) by National Research Foundation of Korea (NRF) to work at Konkuk University. This research was sup-ported by Basic Science Research Program through the NRF funded by the Ministry of Science, ICT \& Future Planning (NRF2020H1D3A2A01060467, NRF-2019R1C1C11009766). This work was also supported by KU Research Professor program of Konkuk University. This paper was supported by Konkuk University Researcher Fund in 2019.”
The original article can be found online at https:// doi.org/10.1007/s12088-020-00883-6.
Vipin Chandra Kalia vckaliaku@gmail.com

$\bowtie$ Jung-Kul Lee jkrhee@konkuk.ac.kr

1 Department of Chemical Engineering, Konkuk University, Seoul 05029, Republic of Korea 